Int. J. Contemp. Math. Sciences, Vol. 2, 2007, no. 5, $241-244$

\title{
Plane Curves with Ordinary Multiple Points and Variables Ordinary Nodes
}

\author{
E. Ballico ${ }^{1}$ \\ Dept. of Mathematics \\ University of Trento \\ 38050 Povo (TN), Italy \\ ballico@science.unitn.it
}

\begin{abstract}
Fix integers $s>0, m_{i}>0,1 \leq i \leq s, d>0, z \geq 0$, and $s$ distinct points of $\mathbf{P}^{2}$. Let $W\left(d ; m_{1} P_{1}, \ldots, m_{s} P_{s} ; z\right)$ denote the set of all integral degree $d$ plane curves with an ordinary point with multiplicity $m_{i}$ at each $P_{i}$ and $z$ ordinary nodes in $\mathbf{P}^{2} \backslash\left\{P_{1}, \ldots, P_{s}\right\}$ as its only singularities. Here we show under certain assumptions (mainly if either $s \leq 5$ or $z \gg 0$ ) that $W\left(d ; m_{1} P_{1}, \ldots, m_{s} P_{s} ; z\right)$ and with the expected dimension.
\end{abstract}

\section{Mathematics Subject Classification: 14H50}

Keywords: plane curve, singular plane curve, ordinary multiple point

Fix integers $s>0, m_{i}>0,1 \leq i \leq s, d>0, z \geq 0$, and $s$ distinct points of $\mathbf{P}^{2}$. Let $W\left(d ; m_{1} P_{1}, \ldots, m_{s} P_{s} ; z\right)$ denote the set of all integral degree $d$ plane curves with an ordinary point with multiplicity $m_{i}$ at each $P_{i}$ and $z$ ordinary nodes in $\mathbf{P}^{2} \backslash\left\{P_{1}, \ldots, P_{s}\right\}$ as its only singularities. Thus each $C \in$ $W\left(d ; m_{1} P_{1}, \ldots, m_{s} P_{s} ; z\right)$ has geometric genus $(d-1)(d-2) / 2-\sum_{i=1}^{s}\left(\begin{array}{c}m_{i} \\ 2\end{array}\right)-z$. Let $u: M \rightarrow \mathbf{P}^{2}$ be the blowing up of $\left\{P_{1}, \ldots, P_{s}\right\}$. Set $E_{i}:=\pi^{-1}\left(P_{i}\right)$ and $L_{d}:=u^{*}\left(\mathcal{O}_{\mathbf{P}^{2}}(d)\right)\left(-m_{1} E_{1}-\cdots-m_{s} E_{s}\right)$. Let $V(M, d, z)$ denote the set of all $C \in\left|L_{d}\right|$ which are integral and with $z$ ordinary points (none of them on $E_{1} \cup \cdots \cup E_{s}$ ) as its only singularities. The strict transform induces an insomorphim between $W\left(d ; m_{1} P_{1}, \ldots, m_{s} P_{s} ; z\right)$ and $V(M, d, z)$.

Notice that either $W\left(d ; m_{1} P_{1}, \ldots, m_{s} P_{s} ; 0\right)=\emptyset$ or $W\left(d ; m_{1} P_{1}, \ldots, m_{s} P_{s} ; 0\right)$ is irreducible and rational. We will say that an irreducible component $\Gamma$ of the

\footnotetext{
${ }^{1}$ The author was partially supported by MIUR and GNSAGA of INdAM (Italy).
} 
scheme $W\left(d ; m_{1} P_{1}, \ldots, m_{s} P_{s} ; z\right)$ has the expected dimension if $\Gamma \neq \emptyset$ and

$$
\operatorname{dim}(\Gamma)=\left(d^{2}+3 d\right) / 2-\sum_{i=1}^{s} m_{i}\left(m_{i}+1\right) / 2-z
$$

Here we state our main results.

Theorem 1. Assume $2 \leq s \leq 5, m_{1} \geq \cdots \geq m-s>0$ and that no 3 of the points $P_{1}, \ldots, P_{s}$ are collinear. If $s=2,3,4$ assume $d \geq m_{1}+m_{2}$. If $s=5$ assume $d \geq m_{1}+m_{2}$ and $m_{2} \geq m_{4}+m+5$. Fix any integer $z$ such that $0 \leq z \leq\left(d^{2}+3 d\right) / 2-\sum_{i=1}^{s} m_{i}\left(m_{i}+1\right) / 2$. Then $W\left(d ; m_{1} P_{1}, \ldots, m_{s} P_{s} ; z\right)$ is non-empty and it has an irreducible component with the expected dimension.

Theorem 2. Assume $d \geq \sum_{i=1} m_{i}$ and fix any integer $z$ such that $0 \leq z \leq$ $\left(d^{2}+3 d\right) / 2-\sum_{i=1}^{s} m_{i}\left(m_{i}+1\right) / 2$. Then $W\left(d ; m_{1} P_{1}, \ldots, m_{s} P_{s} ; z\right)$ is non-empty and it has an irreducible component with the expected dimension.

Remark 1. Here we assume $1 \leq s \leq 8$ and that $\left(P_{1}, \ldots, P_{s}\right)$ is general in $\left(\mathbf{P}^{2}\right)^{s}$. Hence $K_{M}^{*}$ is ample. Thus $K_{M} \cdot T<0$ for all integral curves $T \subset M$. We will always assume $m_{1} \geq \cdots \geq m_{s}>0$. Here we will only do the case $z=0$. For the case $z>0$, see Remark 2. Since the case $s=1$ was done in [1], we will assume $2 \leq s \leq 8$. In all cases (under suitable assumpitions of the integers $d$ and $m_{i}, 1 l e i \leq s$, we will show that $W\left(d ; m_{1} P_{1}, \ldots, m_{s} P_{s} ; 0\right)$ is non-empty and with the expected dimension given by (1).

(a) Here we assume $s=2$. We also assume $d \geq m_{1}+m_{2}$. Let $E \subset \mathbf{P}^{2}$ a general union of $m_{2}$ smooth conics through $P_{1}$ and $P_{2}, m_{1}-m_{2}$ lines through $P_{1}$ and (if $d>m_{1}+m_{2}$ ) a general union of $d-m_{1}-m_{2}$ lines. Let $F \subset M$ the strict transform of $E$ in $M$. Notice that $F$ is reduced and connected. A general $X \in|F|$ is smooth and connected, and $W\left(d ; m_{1} P_{1}, m_{2} P_{2} ; 0\right)$ has the expected dimension ([1], Prop. 3.7, [4], Lemma 2 and Prop. 2.11, and [2], Lemma 3).

(b) Here we assume $s=3$. We also assume $d \geq m_{1}+m_{2}$. Let $E$ be the general union of $m_{3}$ smooth conics containing $P_{1}, P_{2}, P_{3}, m_{2}-m_{3}$ smooth conics through $P_{1}$ and $P_{2}, m_{1}-m_{2}$ lines through $P_{1}$ and (if $d>m_{1}+m_{2}$ ) a union of $d-m_{1}-m_{2}$ lines. Let $F$ be the strict stransform of $E$ in $M$. $F$ is reduced and connected. A general $X \in|F|$ is smooth and connected, and $W\left(d ; m_{1} P_{1}, m_{2} P_{2} ; 0\right)$ has the expected dimension ([1], Prop. 3.7, [4], Lemma 2 and Prop. 2.11, and [2], Lemma 3).

(c) Here we assume $s=4$. We also assume $d \geq m_{1}+m_{2}$. Let $E$ be the general union of $m_{4}$ smooth conics containing $P_{1}, P_{2}, P_{3}, P_{4}, m_{3}-m_{4}$ smooth conics containing $P_{1}, P_{2}, P_{3}, m_{1}-m_{3}$ lines through $P_{1}, m_{2}-m_{3}$ lines through $P_{2}$ and (if $d>m_{1}+m_{2}$ ) a general union of $d-m_{1}-m_{2}$ lines. Let $F$ be the strict transform of $E$ in $M . F$ is reduced and connected. A general $X \in|F|$ is smooth and connected, and $W\left(d ; m_{1} P_{1}, m_{2} P_{2}, m_{3} P_{3} ; 0\right)$ has the expected dimension ([1], Prop. 3.7, [4], Lemma 2 and Prop. 2.11, and [2], Lemma 3). From no on, we omit to mention that $F \subset M$ is the strict transform of $E$ and to quote [1], Prop. 3.7, [4], Lemma 2 and Prop. 2.11, and [2], Lemma 3. 
(d) Here we assume $s=5$. First assume $m_{3} \geq m_{4}+m_{5}$ and $d \geq m_{1}+m_{2}$. Let $E$ be the general union of $m_{4}$ smooth conics through $P_{1}, P_{2}, P_{3}, P_{4}, m_{5}$ smooth conics through $P_{1}, P_{2}, P_{3}, P_{5}, m_{3}-m_{4}-m_{5}$ smooth conics through $P_{1}, P_{2}, P_{3}, m_{1}-m_{3}$ lines through $P_{1}, m_{2}$ lines through $P_{2}$ and (if $d>m_{1}+m_{2}$ ) a general union of $d-m_{1}-m_{2}$ lines. Now assume $m_{2} \geq m_{4}+m_{5}, m_{3}<$ $m_{4}+m_{5}$, and $d \geq m_{1}+m_{2}$. Let $E$ be a general union of $m_{5}$ smooth conics through $P_{1}, P_{2}, P_{3}, P_{5}, m_{4}+m_{5}-m_{3}$ smooth conics through $P_{1}, P_{2}, P_{3}, P_{4}$, $m_{3}-m_{5}$ smooth conics through $P_{1}, P_{2}, P_{4}, m_{1}-m_{4}-m_{5}$ lines through $P_{1}$, $m_{2}-m_{4}-m_{5}$ lines through $P_{2}$ and (if $d>m_{1}+m_{2}$ ) a general plane curve of degree $d-m_{1}-m_{2}$.

(e) Here we assume $s=6$. Assume $d \geq 2\left(m_{1}+m_{2}+m_{3}\right)$. By [3], p. 303, $W\left(d ; m_{1} P_{1}, \ldots, m_{s} P_{s} ; 0\right) \neq \emptyset$ and with the expected dimension.

(f) Here we assume $s=7$. Assume $d \geq 2\left(m_{1}+m_{2}+m_{3}\right)+m_{7}$. Take a general solution $C^{\prime}$ of the case $s^{\prime}:=6, d^{\prime}:=d-m_{7}, m_{i}^{\prime}:=m_{i}$ for $1 \leq i \leq 6$ (part (e)). We may assume $P_{7} \notin C^{\prime}$. Let $E$ be the union of $C^{\prime}$ and $m_{7}$ general lines through $P_{7}$. Call $C^{\prime \prime} \subset M$ the strict transform of $C^{\prime}$. Since $K_{M} \cdot C^{\prime \prime}<0$, we get that $|F|$ contains a smooth and connected element. Hence $W\left(d ; m_{1} P_{1}, m_{2} P_{2} ; 0\right)$ is not empty and it has the expected dimension.

(g) Here we assume $s=8$. Assume $d \geq 2\left(m_{1}+m_{2}+m_{3}\right)+m_{7}+m_{8}$. Take a general solution $C^{\prime}$ of the case $s^{\prime}:=6, d^{\prime}:=d-m_{7}-m_{8}, m_{i}^{\prime}:=m_{i}$ for $1 \leq i \leq 6$ (part (e)). We may assume $P_{i} \notin C^{\prime}$ for $i=7,8$. Let $E$ be the union of $C^{\prime}, m_{8}$ general smooth conics containing $P_{7}$ and $P_{8}$ and (if $m_{7}>m_{8}$ ) $m_{7}-m_{8}$ general lines through $P_{7}$. Call $C^{\prime \prime} \subset M$ the strict transform of $C^{\prime}$. Since $K_{M} \cdot C^{\prime \prime}<0$, we get that $|F|$ contains a smooth and connected element. Hence $W\left(d ; m_{1} P_{1}, m_{2} P_{2} ; 0\right)$ is not empty and it has the expected dimension.

Remark 2. Here we assume $1 \leq s \leq 8$ and that $\left(P_{1}, \ldots, P_{s}\right)$ is general in $\left(\mathbf{P}^{2}\right)^{s}$. Hence $K_{M}^{*}$ is ample. Thus $K_{M} \cdot T<0$ for all integral curves $T \subset M$. We will always assume $m_{1} \geq \cdots \geq m_{s}>0$. Here we will assume $z>0$, see Remark 2. Since the case $s=1$ was done in [1], we will assume $2 \leq s \leq 8$. In all cases (under suitable assumpitions of the integers $d$ and $m_{i}, 1 l e i \leq s$, we will show the existemce of an irreducible component $\Gamma$ of $W\left(d ; m_{1} P_{1}, \ldots, m_{s} P_{s} ; z\right)$ is non-empty and with the expected dimension given by (1). We will take the set-up of Remark 1. In each case we have a reducible degree $d$ plane curve $E$ and its transform $F$ of $E$ in $M$. We fix $z$ suitable singular points of $F$, say $O_{1}, \ldots, O_{z}$ and we call $M^{\prime}$ the blowing-up of $M$ at these points. Notice that $M^{\prime}$ depends from the choice of $E$ and then from the choice of $A:=$ $\left\{O_{1}, \ldots, O_{z}\right\}$. Let $G$ be the strict transform of $F$ in $M^{\prime}$. Hence $G$ is reduced. The set $A$ is said to be a non-disconnecting set if $G$ is connected ([4]). We will always choose $A$ so that $G$ is connected. This is the only condition we need to apply [4], Lemma 2 and Prop. 2.11, and get the existence of an irreducible component of $W\left(d ; m_{1} P_{1}, \ldots, m_{s} P_{s} ; z\right)$ with the expected dimension. In each case below let $c_{F}$ denote the number of the irreducible components of $F$. In parts (a) each irreducible component of $F$ is a smooth rational curve and 
$p_{a}(F)=\left(d^{2}+3 d\right) / 2-\sum_{i=1}^{s} m_{i}\left(m_{i}+1\right) / 2$. Hence in these cases $F$ has $\left(d^{2}+\right.$ $3 d) / 2-\sum_{i=1}^{s} m_{i}\left(m_{i}+1\right) / 2+c_{F}-1$ singular points. Notice that we may find a non-disconnecting subset of $\operatorname{Sing}(F)$ with cardinality $\left(d^{2}+3 d\right) / 2-$ $\sum_{i=1}^{s} m_{i}\left(m_{i}+1\right) / 2$. Hence in these cases we may tale as $z$ any integer such that $0 \leq z \leq\left(d^{2}+3 d\right) / 2-\sum_{i=1}^{s} m_{i}\left(m_{i}+1\right) / 2$.

(a) Here we asume $s=2$. Use the construction of part (a) of Remark 1 .

We win if $d \geq m_{1}+m_{2}$ and $0 \leq z \leq\left(d^{2}+3 d\right) / 2-\sum_{i=1}^{2} m_{i}\left(m_{i}+1\right) / 2$.

(b) Here we assume $s=3$. Use the construction of part (b) of Remark 1 .

We win if $d \geq m_{1}+m_{2}$ and $0 \leq z \leq\left(d^{2}+3 d\right) / 2-\sum_{i=1}^{3} m_{i}\left(m_{i}+1\right) / 2$.

(c) Here we assume $s=4$. Use the construction of part (c) of Remark 1 .

We win if $d \geq m_{1}+m_{2}$ and $0 \leq z \leq\left(d^{2}+3 d\right) / 2-\sum_{i=1}^{4} m_{i}\left(m_{i}+1\right) / 2$.

(d) Here we assume $s=5$. Use the construction of part (a) of Remark 1. We win if $d \geq m_{1}+m_{2}$ and $m_{2} \geq m_{3}+m_{5}$ and $0 \leq z \leq\left(d^{2}+3 d\right) / 2-$ $\sum_{i=1}^{2} m_{i}\left(m_{i}+1\right) / 2$.

Proof of Theorem 1. All the statements were proved in Remark 2.

Proof of Theorem 2. Let $E$ be the general union of $m_{i}$ lines through $P_{i}$ for all $1 \leq i \leq s$ and (if $d>\sum_{i=1} m_{i}$ ) of $d-\sum_{i=1} m_{i}$ lines. Then use the first part of Remark 2.

We work over an algebraically closed field $\mathbb{K}$ such that $\operatorname{char}(\mathbb{K})=0$.

\section{REFERENCES}

[1] E. Arbarello and M. Cornalba, Footnotes to a paper of Beniamino Segre, Math. Ann. 256 (1981), 341-362.

[2] M. Coppens, The Weierstrass gap sequence of the ordinary ramification points of trigonal coverings of $\mathbf{P}^{1}$, J. Pure Appl. Algebra 43 (1986), no. 1, 11-25.

[3] R. Hartshorne, Genre des courbes algébriques dans l'espace projectif, Sem. Bourbaki 1981/82, no. 592, Astérisque 92-93 (1992), 301-313.

[4] A. Tannenbaum, Families of algebraic curves with nodes, Compositio Math. 41 (1980), no. $1,107-126$.

Received: August 23, 2006 